\title{
Occurrence of Floating Toe from the Viewpoint of the Structure of Foot Arch
}

\author{
KATSUHIKO FUKUYAMA ${ }^{1,2)}$, HitOSHI MARUYAMA ${ }^{3)}$ \\ ${ }^{1)}$ Department of Physical Therapy Faculty of Medical and Health Science, Tsukuba International \\ University: 6-8-33 Manabe Tsuchiura Ibaraki 300-0051 Japan. \\ TEL: +8129-826-6622,E-mail: k-gtf@canlenet.ne.jp \\ 2) Physical Therapy Section, Health Science Program, Health and Welfare Science Course, Graduate \\ School of International University of Health and Welfare \\ ${ }^{3)}$ Department of Physical Therapy, Faculty of Health Science, International University of Health and \\ Welfare
}

\begin{abstract}
Purpose] The purpose of this research was to investigate the occurrence and mechanism of floating toe from the viewpoint of the structure of foot arch.[Subjects and Methods] The subjects were 65 adult females who had no history of orthopedic disease. Subjects stood on a pedoscope and we took images of the soles. According to a floatingtoe score which we created, we allocated subjects to a normal group and a floating-toe group using the obtained images. We measured the arch height percentage in the sitting position and standing position in the normal group and floatingtoe group and found the difference of arch height percentage (value obtained by subtracting the arch height percentage in the standing position from that in the sitting position). Results of the measurements were compared between the two groups. [Results] Among 65 cases, 23 were allocated to the normal group and 18 were allocated to the floating-toe group. There was no significant difference between the two groups for the arch height percentage in the sitting and standing positions. The difference of arch height percentage in the floating-toe group was significantly smaller. [Conclusion] We presume that there are two types of foot - the type in which the arch rises and the type in which the arch lowers in the floating toe, and lifestyle, footwear and other factors are involved.
\end{abstract}

Key words: Floating toe, Image of sole, Arch height percentage

(This article was submitted May 11, 2010, and was accepted Jun. 21, 2010)

\section{INTRODUCTION}

The foot of a human has to support the weight, absorb impact, and push the body forward (as impelling force). For humans who graduated to bipedal walking from quadrupedal walking, the function of the forepart of the foot is extremely important for standing firmly on the ground with both feet, stabilizing the body, and walking and running.

Recently, "Floating toe" has become a problem of the forepart of the foot. We define floating toe as a condition in which the toe does not contact the ground in the standing position and the weight does not shift to the toe in walking. We previously reported that, toe-grip strength and capability to shift the center of gravity forward decline due to weakening of toe flexors, and motion adjustment ability, including the sensory factors which are required for the execution of more accurate motion, also declines in cases of floating toe ${ }^{1)}$. Especially, with regard to sensory factors, we think that disruption of information input from the floor, and reduction in the capacity and degeneration of mechanoreceptor, is related to floating toe. Accordingly, we presume that floating toe influences balance, walking and other functions.

Some research the relationship between floating toe and motor function conducted by others, and their results can be summarized as follows. In cases of floating toe, the force maintaining toe pressure weakens. This does not influence the static balance greatly, but it does influence the dynamic balance significantly, and it is also related to the length of stride and walking speed ${ }^{2-5)}$.

As for the cause of floating toe, the aftereffect of surgical operation and natural occurrence are reported. For example, Migues $^{6)}$, Hofstaetter ${ }^{7)}$ and others point out that floating toe occurs as an aftereffect of Weil osteotomy, a surgical operation on the foot. As for weakening of the natural occurrence, a relationship with hypoplasia of the foot arch due to muscle related to the formation of the medial arch has been reported ${ }^{8,9)}$. The muscles of the forepart of the foot, the muscles related to the action of the toes as an impelling force, are the toe flexor and triceps sural muscles. To efficiently fulfill their functions, the functions of the plantar fascia, toe extensor, abductor halluces muscle, posterior tibial muscle and peroneus, which are related to the formation of foot medial arch, are also important. Especially, it has been reported that floating toe occurs more 

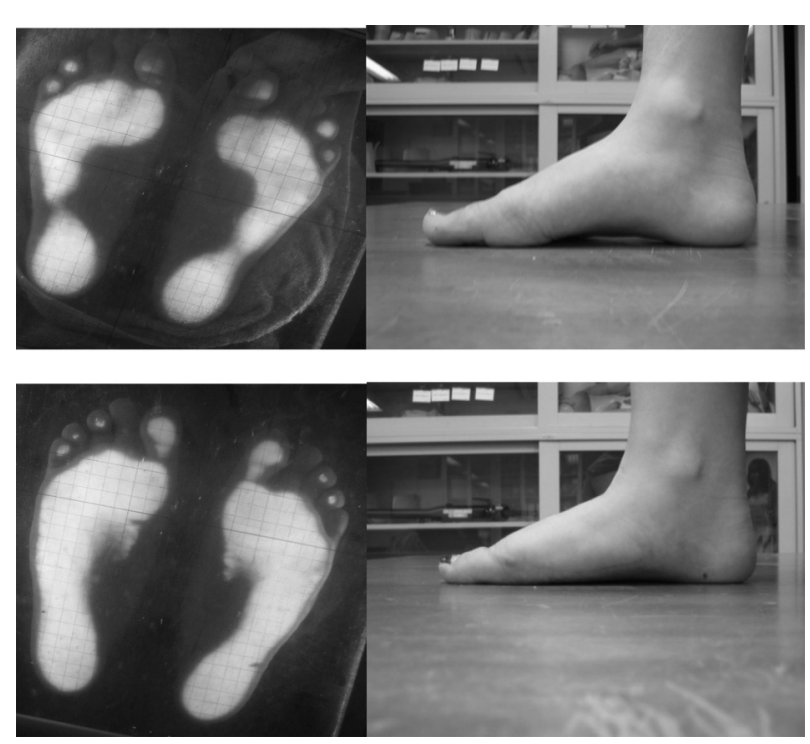

Fig. 1. Types of floating toe in which the arch rose (top) and the arch lowered (bottom).

often in the outer toes than in the big toe, and it is thought that this is because of pronation of the foot with flattening of the medial $\operatorname{arch}^{10)}$. Because of the reports, we initially thought that floating toe was a condition in which lowering of the medial arch occurred due to decrease of tension in the toe flexor muscles. However, when we examine Pedoscope images of floating toe cases, we found not only images of medial arch lowering, so-called flat foot, but also many in which the medial arch was intact (Fig.1). In other words, We think that there are at least two types of floating toe.

Accordingly, in this research, we aimed to investigate the occurrence and mechanism of floating toe based on the relationship between floating-toe and arch structure by taking images of soles with Pedoscope and measuring the arch height percentage.

\section{SUBJECTS AND METHODS}

\section{Subjects}

The subjects were 65 healthy adult female who had no history of orthopedic disease in the legs. Their mean age was $22.1 \pm 2.1$ years old. Prior to conducting our research, we explained the purpose and contents of the research and the handling of data to all the subjects and obtained their written consent.

\section{Methods}

First, we captured images of the soles of the 65 subjects with our custom-built Pedoscope. Subjects were instructed to stand upright on the stage of hardened glass on the Pedoscope with the feet $5 \mathrm{~cm}$ apart and to gaze at a target placed $2 \mathrm{~m}$ ahead and on the level while keeping an easy and natural standing position. We captured images of the sole in contact with the glass plate using a digital camera and a reflecting mirror.

Based on the obtained images, we calculated the "floating-toe score", using our original criteria ${ }^{11)}$. For the 10 toes of both feet, if a toe appeared clearly on the image, 2 points were given, if it appeared unclearly, point 1 was given, and if it did not appear, no points were given; points were summed to realize the floating-toe score.

Cases in which both big toes were given 2 points and the floating-toe score was more than 18 points were allocated to the normal group, and cases in which the floating-toe score was less than 10 points were allocated to the floating-toe, group. In this way, the two groups were determined.

Next, the arch height percentage of both of these groups as calculated. To find the arch height percentage, we divided the navicular bone height by the foot length in chair sitting and static standing and expressed the results as percentages. In chair sitting, we instructed the subject to position the trunk against the back of the chair, keep the knee joint flexed at $90^{\circ}$, and place the soles in contact with the floor without applying a load. In static standing, we instructed the subjects to stand on a working table level with the floor with their feet $10 \mathrm{~cm}$ apart and gaze at a target placed $2 \mathrm{~m}$ ahead on the level . Marking the rough surface of the navicular bone and the first metatarsal head, we measured the height from the floor to the raised area (height of the navicular bone) and the length from the posterior surface of the calcaneus to the first metatarsal head (foot length) with a ruler. We obtained the arch height percentage by dividing the height of navicular bone by the foot length. We gained individual arch height percentages in the sitting position and static standing, and the difference of arch height percentage was obtained by subtracting the arch height percentage in the standing position from that in the sitting position.

We compared the arch height percentage in the sitting position with that in standing position and also the difference of arch height percentage of the normal group with that of the floating-toe group using the Mann-Whitney U test. Statistical work, statistical analysis software, SPSS $11.5 \mathrm{~J}$ for the statistical analyses, and $\mathrm{p}$ values less then $5 \%$ were considered significant.

\section{RESULTS}

Using the floating-toe score based on the sole images of 65 subjects, 23 cases were allocated to the normal group and 18 cases were allocated to the floating-toe group. Among the 24 cases which did not belong to either group, there were 5 cases which were not included in the normal group due to insufficient contact of the big toe with the floor even though they had a floating-toe score of more than 18 points.

The arch height percentage in the sitting position and the standing position and the difference of arch height percentage in the two groups are shown in Table 1. The arch height percentage in the sitting position was $21.23 \pm 1.59 \%$ in the normal group and $20.88 \pm 2.72 \%$ in the floating-toe group with no significant difference between the groups. The arch height percentage in the standing position was $18.47 \pm 1.41 \%$ in the normal group and $18.55 \pm 2.66 \%$ in the floating-toe group with no significant difference between the groups. The difference of arch height percentage was $2.75 \pm 0.67$ in the normal group and $2.03 \pm 0.75$ in the floating-toe group, and the value of the floating toe group 
Table 1. Arch height percentage in the both groups

\begin{tabular}{lcc}
\hline & Normal group & Floating toe group \\
\hline Subjects (n) & 23 & 18 \\
Sitting(\%) & $21.23 \pm 1.59$ & $20.88 \pm 2.72$ \\
Standing(\%) & $18.47 \pm 1.41$ & $18.85 \pm 2.66$ \\
Difference of arch height percentage & $2.75 \pm 0.67$ & $2.03 \pm 0.75^{*}$ \\
\hline
\end{tabular}

Values are mean $\pm \mathrm{SD}$. ${ }^{*} \mathrm{p}<0.05$ between normal group and floating toe group. Impact Factors and projection.

was significantly lower than that of the normal group $(\mathrm{p}<0.05)$.

\section{DISCUSSION}

In the measurement of arch height percentage in the sitting position and the standing position, there was no significant difference between the normal group and the floating-toe group. We think that floating toes exists regardless of the raising or lowering of the arch as the standard deviation was large in the floating-toe group.

It is thought that floating toe (the type where the arch rose) is caused by wearing too small shoes and high-heeled shoes. Kapandji ${ }^{12)}$ says that the foot slides forward due to the inclination of shoe, the toe contacts with the top of shoe and extends, and as a result, the mark of toe is not left. Hyperextension of the toe lowers the head of the metatarsal, and the continuation of this state increases the tension of toe extensors. The plantar fascia is pulled forward, the heel comes close to the toe and the arch rises due to the tension of the toe extensors. We think that this is one cause of floating toe (the type in which the arch rises).

In contrast, in the type in which the arch is lowered, we think that the arch lowers due to the decrease of the force of the muscles associated with the arch formation, such as toe flexor muscle, abductor hallucis muscle, posterior tibial muscle and others, and in this case, lowering of the transverse arch in the forepart of the foot and hallux valgus occurs. Onuki and others ${ }^{13)}$ focused on the difference of childcare conditions of 5-year-old children, and assessed an indoor play group and outdoor play group using footprints and photographs in relation to the average amount of time of outdoor play in a year. They reported that the incidence of floating-toe and flat feet was significantly higher in the indoor play group. Harada and others ${ }^{14)}$ reported that floating-toe had clearly increased and the height of the foot arch had decreased drastically as compared with 20 years earlier, and they identified one associated factor as the method of transport to kindergarten. These reports have given rise to the belief that floating toe in which the arch lowers with lifestyle factors such as outdoor play, play in bare feet, and use of vehicles.

We examined floating toe using the difference of arch height percentage. As the difference of arch height percentage in the floating-toe group was significantly smaller, we think that the lowering of the arch under load is less in the floating-toe group than in the normal group, since it is found in floating-toe of both types (arch lowered or arch raised). Possible causes are as follows: In the lowered arch type, as the arch is not formed sufficiently, there is little difference even though the arch lowers under load. In the raised arch type, the tension of toe extensor and plantar fascia muscles reduces the flexibility of the foot, so it does not lower sufficiently gripping even when load is applied. Murata and others ${ }^{15)}$ investigated the factors involved in the toe holding force, which decreases in the case of floatingtoe, and, identified three associated factors, arch height percentage, flexibility of foot and weight. The flexibility of foot is evaluated by subtracting the length when the forepart of foot is bent at a maximum from the foot length (length from heel to toe) in static standing. Based on Murata's report, we think that the distance between the heel and the forepart of foot is shortened due to shrinking of the plantar fascia and toe extensor muscles in the raised arch type of floating toe, and the reduction of foot flexibility is related to the reduction of toe gripping force. Also, in the lowered arch type of floating toe, we think that weakening of the muscle group which forms the arch itself is related to the decrease of toe gripping force. However, in the present study, there was an insufficient number of cases for classifying into raised arch and lowered arch types of floating-toe groups, and since the difference of arch height percentage varied widely, it casts doubt on.

It is thought that various lifestyle factors are involved in the occurrence of floating toe. In the future, it will be necessary to conduct further investigations additionally examining the factors such as play in childhood, experience of sports, and footwear numbers of floating toe cases as well as classifying them into raised and lowered arch type groups.

\section{REFERENCES}

1) Fukuyama $\mathrm{K}$, Osanai M, Maruyama $\mathrm{H}$ : Adult toe contact and the function of floating toes. Rigakuryoho kagaku, 2009, 24: 683-687.

2) Kabe N, Kurosawa K, Nishida Y, et al.: The study of relationship between toe and dynamic postural control. Rigakuryoho kagaku, 2002, 17: 199-204.

3) Handa S, Horiuchi K, Aoki K: A study on the measurement of toes grasping strength and effect of standing postural control. Ningenn kogaku, 2004, 40: 139-147.

4) Tsujino A, Tanaka N: Toe flexor force and the location of center of pressure in performance of standing reaching tasks. Rigakuryoho kagaku, 2007, 22: 245-248.

5) Fukuda I, Kobayashi R: The effect of the toe grip training in healthy young adults. Rigaku ryouhogaku, 2008, 35: 261-266.

6) Miguis A, Slullitel G, Bilbao F, et al.: Floating toe deformity as a complication of the weil osteotomy. Foot Ancle Int, 2004, 25: 609-613.

7) Hofstaetter SG, Hofstaetter JG, Petroutsas JA, et al.: The weil osteotomy: A seven year follow up. J Bone Joint Surg, 2005, 87: 1507-1512.

8) Onuki N, Washida $T$, Narita $M$, et al.: Characteristics of infant's floatage 
toes. OT Jounal, 2005, 39: 261-268.

9) Tsuneya M, Usui N: Actual state of toe contact in the upright position among healthy adults. Rigaku ryohogaku, 2006, 33: 30-37.

10) Uchida T, Fuziwara K, Sasaki K, et al.: Measurement of foot configuration of kindergarten children. Kutsuno igaku, 2002, 16: 96-99.

11) Yahagi $T$, Nemoto K, Fukuyama K: Utilizing a zouri to treat a floating toes and ease low back pain. Kutsuno igaku, 2004, 18: 65-71.

12) Kapandji IA: Physiologie articulaire II. Tokyo, Ishiyaku syuppan, 1998, pp238-239.

13) Onuki N, Washida T, Narita M, et al.: Comparison of children's outdoor play hours and occurrence of floating toe. Sagyo ryoho, 2005, 24: 461-473.

14) Harada S: A study on physical structures of preschool children's feet compared between 1980 and 2000. Kutsuno igaku, 2001, 15: 14-18.

15) Murata $S$, Kutsuna $T$ : Impact factors and projection of foot-gripping force. Rigakuryoho kagaku, 2003, 18: 207-212. 